\title{
Elementary symmetric functions of two solvents of a quadratic matrix equation
}

\author{
M A Jivulescu ${ }^{1,2}$, A Napoli $^{1}$, A Messina $^{1}$ \\ ${ }^{1}$ MIUR, CNISM and Dipartimento di Scienze Fisiche ed Astronomiche, \\ Università di Palermo, via Archirafi 36, 90123 Palermo, Italy \\ 2 Department of Mathematics, " Politehnica" University of Timişoara, \\ P-ta Victoriei Nr. 2, 300006 Timişoara, Romania \\ Email Address:1,2 maria.jivulescu@mat.upt.ro \\ Email Address: ${ }^{1}$ messina@fisica.unipa.it
}

September 18, 2021

\begin{abstract}
Quadratic matrix equations occur in a variety of applications. In this paper we introduce new permutationally invariant functions of two solvents of the $n \times n$ quadratic matrix equation $X^{2}-\mathcal{L}_{1} X-$ $\mathcal{L}_{0}=0$, playing the role of the two elementary symmetric functions of the two roots of a quadratic scalar equation. Our results rely on the connection existing between the QME and the theory of linear second order difference equations with noncommutative coefficients. An application of our results to a simple physical problem is briefly discussed.
\end{abstract}

Keywords: quadratic matrix equation; solvent; difference equation; symmetric functions.

\section{INTRODUCTION}

Matrix language belongs to all Sciences where multicomponent variables and noncommutativity enter scene in the description of the system under scrutiny. In such situations matrix methods allow indeed compact formulations and elegant resolutions of linear and sometimes nonlinear problems. As an example, consider the time development of a quantum system investigated in the Heisenberg picture. One is in general faced with a matrix, often nonlinear, systems of differential equations typically very difficult to handle due to the noncommutativity of the involved observables. Even when the problem under investigation enables the decoupling of this system achieving 
linear differential Heisenberg equations in a $n$-dimensional Hilbert space, the application of the characteristic equation method, so useful to write down the general integral in the scalar case, originates a $n$-dimensional matrix nonlinear algebraic equation whose resolution cannot unfortunately rely on general propositions. Differently from the case $n=1$ (that is the scalar case), the fundamental theorem of algebra does not indeed hold, so that the roots (usually called solvents) of an algebraic matrix equation may exist or not and even stipulating their existence, their number cannot be simply related to the degree of the equation and could in particular be infinite. It is thus not surprising that the theory of algebraic matrix equations both for its wide applicability and as research subject aiming extending the beautiful chapter of the scalar algebraic equations, has recently received a great deal of attention in the mathematical literature (Gantmacher 1998, Gohberg 2006, Horn 1999). Here we recall the analysis of the spectral properties of the associated matrix pencil via that of the matrix equation itself (Gohberg et al. 1978, Krein et al. 1978). Many research papers on the properties of a quadratic matrix equation, especially numerical approaches, have appeared over the last years (Bai et al. 2005, Butler et all 1985, Dennis et all 1978, Highham 1987, 2000, 2001, Shurbet et al 1974).

Our paper investigates the second degree $n$-dimensional matrix algebraic equations possessing the following canonical form

$$
X^{2}-\mathcal{L}_{1} X-\mathcal{L}_{0}=0
$$

where the unknown $X$ and the two, generally not commuting, coefficients $\mathcal{L}_{0}, \mathcal{L}_{1}$ belong to $M_{n}(\mathbb{C})$, the algebra of all complex square matrices of order $n$. We call this equation a right quadratic matrix equation (RQME). Its resolution is far from being trivial essentially because the simple resolutive formula holding for the quadratic scalar equation (QSE) is not in general applicable due to the noncommutative character of the problem.

Here right means that the linear term in eq. (1) has the form $\mathcal{L}_{1} X$ instead of $X \mathcal{L}_{1}$ or $\mathcal{L}_{1} X+X \mathcal{L}_{1}^{\prime}$ defining a left (LQME) or a bilateral quadratic matrix equation (BQME) respectively. Right, left and bilateral matrix equations are particular cases of the Riccati algebraic matrix equation (Hore et al. 1999) whose canonical form is

$$
X A X+B X+X C+D=0
$$

In the next section we will show that the resolution of this equation is always traceable back to that of a related RQME.

Thus, in this paper we concentrate on the properties possessed by eq. (11) and explore whether or at which extent they may be thought of as generalizations of well-known properties of elementary QSE. In this spirit we seek analogies and differences between the two quadratic equations, matrix and scalar, and, in connection with eq. (11), we formulate the following questions: 
Q1) To find the number of its solvents;

Q2) To express its solvents, if any, in terms of its coefficients and viceversa;

Q3) To define symmetric functions of a pair of solvents.

It is well known that all three questions may be satisfactorily coped with when the quadratic equation is the scalar one. We shall show that the matrix nature of the unknown $X$ of eq. (11) together or not with the noncommutativity between the two coefficients as well as between a solvent and the same $\mathcal{L}_{1}$ and $\mathcal{L}_{0}$, determine properties of eq.(1) with no counterpart among those possessed by the scalar equation. Our investigation will bring to light interesting relations between the solvents of eq. (11) and its coefficients $\mathcal{L}_{1}$ and $\mathcal{L}_{0}$ interpretable as generalizations of the classical Girard-Newton and Waring formulas (Sansone 1952).

In the section 3, devoted to the questions Q1) and Q2), we introduce the notation, give a short sketch of the existing literature and report ad-hoc built examples supporting some theoretical statements. Globally speaking, develop step by step convincing arguments enabling us to introduce, on a heuristic basis, our new definition of elementary functions of two solvents of a quadratic matrix equation.

The main and novel results of this paper are constructed in connection with our reply to Q3) and are reported in section 4 where we introduce and study the properties of the permutationally invariant functions of two solvents of the quadratic matrix equation.

\section{Mapping a Riccati algebraic equation into a RQME}

The Riccati algebraic matrix equation (Hore et al. 1999) in the unknown $Z \in M_{n}(\mathbb{C})$

$$
Z A Z+\widetilde{\widetilde{\mathcal{L}_{1}}} Z+Z \widetilde{\widetilde{\mathcal{L}^{\prime}}}{ }^{1}+\widetilde{\widetilde{\mathcal{L}_{0}}}=0, \quad \widetilde{\widetilde{\mathcal{L}^{\prime}}}{ }_{1}, \widetilde{\widetilde{\mathcal{L}_{1}}}, \widetilde{\widetilde{\mathcal{L}_{0}}} \in M_{n}(\mathbb{C})
$$

assuming the existence of $A^{-1}$ and introducing the change of variable $Z=$ $Y A^{-1}$ may be reduced to the following BQME in $Y$ :

$$
Y^{2}+\widetilde{\mathcal{L}_{1}} Y+Y \widetilde{\mathcal{L}^{\prime}}+\widetilde{\mathcal{L}_{0}}=0
$$

with $\widetilde{\mathcal{L}_{1}}=\widetilde{\widetilde{\mathcal{L}_{1}}}$ and $\widetilde{\mathcal{L}^{\prime}}{ }_{1}=A^{-1} \widetilde{\widetilde{\mathcal{L}^{\prime}}}{ }_{1} A$ and $\widetilde{\mathcal{L}_{0}}=\widetilde{\widetilde{\mathcal{L}_{0}}} A$. It is worth noticing that the BQME (4I), in its most general case, that is when both $\widetilde{\mathcal{L}}_{1} \neq 0$ and $\widetilde{\mathcal{L}}^{\prime}{ }_{1} \neq 0$, is traceable back to the RQME in the canonical form (11) by furtherly changing the variable $Y$ to $X=-\widetilde{\mathcal{L}}^{\prime}{ }_{1}-Y$, where now $\mathcal{L}_{1}=\widetilde{\mathcal{L}_{1}}-\widetilde{\mathcal{L}_{1}^{\prime}}$ and $\mathcal{L}_{0}=\widetilde{\mathcal{L}_{1} \widetilde{\mathcal{L}}_{1}^{\prime}}-\widetilde{\mathcal{L}_{0}}$. The particular case when $\widetilde{\mathcal{L}}_{1}=0$ and $\widetilde{\mathcal{L}}^{\prime}{ }_{1} \neq 0$ transforms the BQME (44) into a LQME which, by applying the above proposed change 
of variable, may be traced back to the RQME (11) with $\mathcal{L}_{1}=-\widetilde{\mathcal{L}_{1}^{\prime}}$ and $\mathcal{L}_{0}=-\widetilde{\mathcal{L}_{0}}$.

In all the cases above considered the original matrix equation and its related RQME equation are simultaneously possible or impossible and when the two sets of solvents are not empty, a biunivocal correspondence may be established.

It is worth noting, in passing, that in the symmetric case of BQME ( $\widetilde{\mathcal{L}_{1}}=\widetilde{\mathcal{L}_{1}^{\prime}}$ ), called the symmetrical bilateral quadratic matrix equation ( SBQME), the related right equation becomes $X^{2}=\widetilde{\mathcal{L}}_{1}^{2}-\widetilde{\mathcal{L}_{0}}$, whose solutions exist if the square root of $\widetilde{\mathcal{L}_{1}^{2}}-\widetilde{\mathcal{L}_{0}}$ exists and are denoted by the matrix multivalued symbol $\sqrt{\widetilde{\mathcal{L}}_{1}^{2}-\widetilde{\mathcal{L}_{0}}}$.

The systematic possibility of transforming LQME, BQME and Riccati algebraic matrix equations into a RQME justify why in this paper we concentrate on the canonical form (1) only, thereafter simply called QME.

\section{$3 \quad$ Matrix versus scalar quadratic equation}

In this section we give our reply to the questions Q1) and Q2) beginning a comparison between a QSE with a QME.

\subsection{On the number of solvents}

The fundamental theorem of algebra due to D'Alembert states that each scalar algebraic equation of degree $n$ in the field $\mathbb{C}$ always admits at least one root in $\mathbb{C}$. This theorem, together with the well-known Ruffini's reminderfactor theorem, implies that the number of solutions of the equation is exactly equal to $n$. The examples reported in Appendix A clearly demonstrate that the fundamental theorem of algebra does not hold for a matrix equation which, in turn, may be impossible, possible with finitely-many or infinitelymany solvents in $M_{n}(\mathbb{C})$.

It is easy to persuade oneself that analogous examples for matrix algebraic equations of higher degree may be given. Thus, we do not have at our disposal a general rule directly involving $\mathcal{L}_{0}$ and $\mathcal{L}_{1}$ to assess conclusions on the number of solvents of a matrix algebraic equation without solving it.

It is of relevance to underline at this point that a resolutive algorithm of a QME does exist(Gantmacher 1998) and stems from a theorem stating that if $X$ is any solution of eq. (11) then $g(X)=0$, where $g(\lambda) \equiv \operatorname{det} Q(\lambda)=$ $\operatorname{det}\left(\lambda^{2} E-\mathcal{L}_{1} \lambda-\mathcal{L}_{0}\right)$ is a polynomial in $\lambda$ of degree $2 n$. The key for the resolution of eq.(1) is to exploit the knowledge of the roots of $g(\lambda)$ associated to eq.(11) and their respective multiplicities to build an appropriate change of the variable $X$ into eq.(1) which has the merit of reducing the QME into a set of independent matrix linear equations in a new matrix 
unknown $T$ which must be nonsingular. From a practical point of view the main bottleneck of such a recipe is of course the determination of all the distinct roots of $g(\lambda)$, even if the conditioned resolution of the independent linear system of $n^{2}$ equations to seek the acceptable matrices $T$ might result quite cumbersome. Details on such a procedure may be found for example in (Gantmacher 1998), where the method is presented for right matrix equations of arbitrary degree. Since it is practically hopeless to give a reply to our questions Q1) and Q2) for a generic QME using the above outlined algorithm, other approaches have been searched to investigate on the existence and the number of solutions of a QME.

We quote the following result due to Dennis (1978)

Theorem 1 If $g(\lambda)$ has $p$ distinct eigenvalues $\left\{\lambda_{i}\right\}_{i=1}^{p}$ with $n \leq p \leq 2 n$ and the corresponding set of $p$ eigenvectors $\left\{v_{i}\right\}_{i=1}^{p}$ satisfies the Haar condition, i.e. every subset of $n$ of them is linearly independent, then the different solvents of the $Q M E$ are exactly $\left(\begin{array}{c}2 n \\ n\end{array}\right)$, if $p=2 n$ and at least $\left(\begin{array}{c}p \\ n\end{array}\right)$, if $n \leq p<2 n$, all by the form

$$
S=W \operatorname{diag}\left(\mu_{i}\right) W^{-1} \quad W=\left[w_{1}, \ldots, w_{n}\right]
$$

where the eigenpairs $\left(\mu_{i}, w_{i}\right)_{i=1}^{n}$ are chosen from among the eigenpairs $\left(\lambda_{i}, w_{i}\right)_{i=1}^{p}$ of $g(\lambda)$.

When $p=n$, the distinctness condition of the eigenvalues is not needed, such that we obtain a sufficient condition for the existence of a solvent

Corollary 1 If $g(\lambda)$ has n linearly independent eigenvectors, then QME has a solvent.

The above method furnishes only diagonalizable solvents and in consequence it can fail to identify all solvents and can even produce no solvents when, in fact, solvents exist.

Some studies on the existence of solvents which avoid the knowledge of the eigensystem $Q(\lambda)$ are known; for example, based on the contraction mapping principle technique it is possible to show that if $\mathcal{L}_{1}$ is a nonsingular matrix and

$$
4\left\|\mathcal{L}_{1}^{-1}\right\|\left\|\mathcal{L}_{1}^{-1} \mathcal{L}_{0}\right\|<1
$$

for any subordinate matrix norm, then at least two solvents exist ( Eisenfeld 1973) (it's worth to note the similarity with the positivity condition of the discriminant for the QSE case). A similar but more restrictive condition was derived by McFarland, Lancaster and Rokne (1977) who, using the Kantorovich theorem on the convergence of Newton method, have derived several sets of sufficient conditions for the existence of a solvent. Moreover, Lancaster 1966, Krein and Lager 1978 have proved that the so called "overdamping condition" is sufficient and ensures the existence of at least two solvents of a QME, called the dominant and minimal solvents. 


\subsection{Solvents and coefficients}

Putting $\mathcal{L}_{1}=0$, eq. (11) becomes $X^{2}=\mathcal{L}_{0}$. Such an equation admits the resolutive formula $X=\sqrt{\mathcal{L}_{0}}$ formally similar to that valid for the QSE, the substantial difference being that now $\sqrt{\mathcal{L}_{0}}$ might not exist making accordingly the equation impossible.

When $\mathcal{L}_{1} \neq 0$, the question of the existence of a resolutive formula becomes doubtful. In fact, it should have the form $X=F\left(\mathcal{L}_{0}, \mathcal{L}_{1}\right)$, with $F$ universal and matrix multivalued function of $\mathcal{L}_{0}$ and $\mathcal{L}_{1}$. Since it must reduce to the well known expression valid in the scalar case, it may be conjectured that $F$ must contain operations like the square root of $\Delta \equiv$ $\mathcal{L}_{1}^{2}+4 \mathcal{L}_{0}$. Since this square root might not exist, one would expect in this case to have to do with an impossible equation. But, the following example shows that this argument is generally false. In fact, the equation $X^{2}+2\left(\begin{array}{cc}1 & 0 \\ 0 & -1\end{array}\right) X-\left(\begin{array}{cc}-1 & 1 \\ 0 & -1\end{array}\right)=0$ possesses the solvent $\left(\begin{array}{cc}-1 & 1 / 2 \\ 0 & 1\end{array}\right)$ even if the square root of the matrix $\Delta=\mathcal{L}_{1}^{2}+4 \mathcal{L}_{0}=4\left(\begin{array}{cc}0 & 1 \\ 0 & 0\end{array}\right)$ does not exist.

One might wonder whether for a QME some aspects characterizing the scalar case such as the commutativity between $\mathcal{L}_{0}$ and $\mathcal{L}_{1}$ and the existence of the square root of $\Delta=\mathcal{L}_{1}^{2}+4 \mathcal{L}_{0}$ are sufficient to represent all the solvents of eq. (11) by means of a sort of "resolutive formula" like $X=\left(\mathcal{L}_{1}+\sqrt{\Delta}\right) / 2$. The reply is negative due to the fact that when a specific realization $\overline{\sqrt{\Delta}}$ of the many-valued symbol $\sqrt{\Delta}$ is not expressible in the form of a polynomial in $\Delta$, it might occur that the commutator $\left[\mathcal{L}_{1}, \overline{\sqrt{\Delta}}\right] \equiv\left(\mathcal{L}_{1} \overline{\sqrt{\Delta}}-\overline{\sqrt{\Delta}} \mathcal{L}_{1}\right)$ does not vanish. Thus, the formula $X=\left(\mathcal{L}_{1}+\sqrt{\Delta}\right) / 2$ cannot be used as a resolutive tool even under the two simplifying assumptions, except when, in addition, $\sqrt{\Delta}$ commutes with $\mathcal{L}_{1}$, which is generally not true. Consider indeed the equation $X^{2}-\mathcal{L}_{1} X+\mathcal{L}_{1}^{2} / 4=0$, where $\mathcal{L}_{0} \equiv-\mathcal{L}_{1}^{2} / 4$ commutes by construction with $\mathcal{L}_{1}$ and $\Delta=0$. Of course the square root of the matrix $\Delta=0$ exists and has infinitely-many realizations that is the nilpotent matrices of index 2 defining the set $\mathcal{N}_{2}^{(n)}$. All these matrices, except the null matrix, cannot of course be expressed in the form of a polynomial in $\Delta(=0)$ and thus we expect that "the resolutive formula " $X=\left(\mathcal{L}_{1}+\sqrt{\Delta}\right) / 2$ does not hold even in this simple case. This means that matrix realizations $\overline{\sqrt{0}}$ of $\sqrt{0}$ not commuting with $\mathcal{L}_{1}$ might exist.

Taking for example $\mathcal{L}_{1}=\left(\begin{array}{ll}1 & 1 \\ 0 & 0\end{array}\right)=\mathcal{L}_{1}^{2}$ and $\overline{\sqrt{0}}=\left(\begin{array}{cc}1 & 1 \\ -1 & -1\end{array}\right) \in \mathcal{N}_{2}^{(2)}$ where

$$
\mathcal{N}_{2}^{(2)} \equiv\left\{N_{2}^{(2)}=\left(\begin{array}{cc}
a & b \\
c & -a
\end{array}\right) / a, b, c \in \mathbb{C}, a^{2}+b c=0\right\}
$$


we easily check that $\left(\mathcal{L}_{1}+\overline{\sqrt{0}}\right) / 2$ is not a solvent of the equation $X^{2}-$ $\left(\begin{array}{ll}1 & 1 \\ 0 & 0\end{array}\right) X+\frac{1}{4}\left(\begin{array}{ll}1 & 1 \\ 0 & 0\end{array}\right)=0$. It is possible to prove that the only solvent of this equation is $\mathcal{L}_{1} / 2$ and that it is obtainable from $\left(\mathcal{L}_{1}+\sqrt{0}\right) / 2$ solely choosing $\sqrt{0}=0$. On contrary, the simple QME $(X-E / 2)^{2}=0$ admits infinitely-many solvents, namely $S=E / 2+N_{2}^{(2)}$, even if once again it has $\Delta=0$. Thus, for this example, the formula $X=\left(\mathcal{L}_{1}+\sqrt{\Delta}\right) / 2$ is valid since $\mathcal{L}_{1}=E$ commutes with every realization of $\sqrt{\Delta}$. Another intriguing example is provided by the equation $X^{2}-\mathcal{L}_{1} X=0$ with $\mathcal{L}_{1}=\left(\begin{array}{ccc}0 & 0 & 0 \\ 0 & 0 & 1 \\ 1 / 2 & 0 & 0\end{array}\right)$ and $\mathcal{L}_{0}=0$. In this case $\left[\mathcal{L}_{1}, \mathcal{L}_{0}\right]=0, \Delta=\mathcal{L}_{1}^{2}=\left(\begin{array}{ccc}0 & 0 & 0 \\ 1 / 2 & 0 & 0 \\ 0 & 0 & 0\end{array}\right)$ and the formula $X=\left(\mathcal{L}_{1}+\sqrt{\Delta}\right) / 2$ does not include the matrix $\left(\begin{array}{lll}0 & 0 & 0 \\ 0 & 0 & 1 \\ 1 & 0 & 0\end{array}\right)$ which, in turn, is a solvent of the equation. This last example is interesting because it shows that the resolutive formula may fail since it does not capture all the solvents of the QME.

Summing up, we conjecture heuristically that a general resolutive formula for QME might not indeed exist. It is however of relevance to observe that when $\sqrt{\Delta}$ exists and $\left[\overline{\sqrt{\Delta}}, \mathcal{L}_{1}\right]=0$, for at least one realization $\overline{\sqrt{\Delta}}$ of $\sqrt{\Delta}$, then the QME admits the solvent $X=\left(\mathcal{L}_{1}+\overline{\sqrt{\Delta}}\right) / 2$.

By the way, it is interesting to note that $\sqrt{\Delta}$ exists when QME (1) admits a solvent $S$ commuting with $\mathcal{L}_{1}$. We may indeed write that $S^{2}-\mathcal{L}_{1} S-$ $\mathcal{L}_{0}=S^{2}-\mathcal{L}_{1} S / 2-S \mathcal{L}_{1} / 2+\mathcal{L}_{1}^{2} / 4-\Delta / 4=\left(S-\mathcal{L}_{1} / 2\right)^{2}-\Delta / 4=0 \Rightarrow$ $\left(S-\mathcal{L}_{1} / 2\right)^{2}=\Delta / 4$ which, by definition, assures that $\Delta$ admits square root.

Reversing our point of view, we concentrate now on establishing whether given two matrices $S_{1}$ and $S_{2} \in M_{n}(\mathbb{C})$, there always exists at least a QME admitting $S_{1}$ and $S_{2}$ as solvents (eventually, among others solvents). We know that in the scalar case this important question has a unique positive reply, that is the knowledge of two roots of a scalar equation determines the coefficients of the equation (putting legitimately its director coefficient equal to 1). What can one say about in the case of QMEs? The reply to this question is more articulated than in the scalar case in the sense that all the possibilities may now take place: the given $S_{1}$ and $S_{2}$ may be solvents of only one determinable QME; no QME could exist simultaneously admitting the two given matrices as solvents or there might exist infinitely-many QME satisfying such a condition (see Appendix B).

In the following a matrix pair $M, N \in M_{n}(\mathbb{C}) \operatorname{such}$ that $\operatorname{det}(M-N) \neq 0$ is called complete, accordingly with the definition introduced by ref. (Krein and Langer 1978) in the context of quadratic operator equations. 
We now may prove the following

Theorem 2 Two matrices $S_{1}$ and $S_{2} \in M_{n}(\mathbb{C})$ are simultaneous solvents of only one $Q M E X^{2}-\mathcal{L}_{1} X-\mathcal{L}_{0}=0$, if and only if the matrix pair $S_{1}$ and $S_{2}$ is complete and the coefficients $\mathcal{L}_{1}$ and $\mathcal{L}_{0}$ may be expressed as

$$
\begin{gathered}
\mathcal{L}_{0}=S_{2}^{2}\left(S_{1}-S_{2}\right)^{-1} S_{1}-S_{1}^{2}\left(S_{1}-S_{2}\right)^{-1} S_{2} \\
\mathcal{L}_{1}=\left(S_{1}^{2}-S_{2}^{2}\right)\left(S_{1}-S_{2}\right)^{-1}
\end{gathered}
$$

Proof:

The existence of a unique QME admitting $S_{1}$ and $S_{2}$ as simultaneous solvents of eq. (11) requires that

$$
\left\{\begin{array}{l}
S_{1}^{2}-\mathcal{L}_{1} S_{1}-\mathcal{L}_{0}=0 \\
S_{2}^{2}-\mathcal{L}_{1} S_{2}-\mathcal{L}_{0}=0
\end{array}\right.
$$

or equivalently that

$$
\left\{\begin{array}{r}
\mathcal{L}_{0}=S_{1}^{2}-\mathcal{L}_{1} S_{1}=S_{2}^{2}-\mathcal{L}_{2} S_{2} \\
S_{1}^{2}-S_{2}^{2}=\mathcal{L}_{1}\left(S_{1}-S_{2}\right)
\end{array}\right.
$$

Regarding the second equation as a matrix linear equation in $\mathcal{L}_{1}$ we know by hypothesis that it has only one solution. This fact necessarily requires that $\operatorname{rank}\left(S_{1}-S_{2}\right)=n$, that is $\left(S_{1}-S_{2}\right)$ is not singular and thus necessarily that

$$
\mathcal{L}_{1}=\left(S_{1}^{2}-S_{2}^{2}\right)\left(S_{1}-S_{2}\right)^{-1}
$$

To determine $\mathcal{L}_{0}$ in the desired form we observe that

$$
\begin{gathered}
\mathcal{L}_{0}=S_{1}^{2}-\left(S_{1}^{2}-S_{2}^{2}\right)\left(S_{1}-S_{2}\right)^{-1} S_{1}= \\
S_{1}^{2}\left(S_{1}-S_{2}\right)^{-1}\left(S_{1}-S_{2}\right)-\left(S_{1}^{2}-S_{2}^{2}\right)\left(S_{1}-S_{2}\right)^{-1} S_{1} \\
=S_{2}^{2}\left(S_{1}-S_{2}\right)^{-1} S_{1}-S_{1}^{2}\left(S_{1}-S_{2}\right)^{-1} S_{2}
\end{gathered}
$$

Reciprocally, if the pair $S_{1}$ and $S_{2}$ is complete and $\mathcal{L}_{0}$ and $\mathcal{L}_{1}$ are given by eqs. (8) and (91) respectively, then it is immediate to see that $S_{1}$ and $S_{2}$ are solvents of the corresponding QME.

If $\left(S_{1}-S_{2}\right)$ is not invertible, then the necessary condition $S_{1}^{2}-S_{2}^{2}=$ $\mathcal{L}_{1}\left(S_{1}-S_{2}\right)$ may be incompatible with the existence of a QME having $S_{1}$ and $S_{2}$ as solvents or may give rise to infinitely-many possibilities for $\mathcal{L}_{1}$ and $\mathcal{L}_{0}$. This means that there might even exist infinitely many QME possessing $S_{1}$ and $S_{2}$ as simultaneous solvents. In the appendix B we give an example of two matrices $S_{1}$ and $S_{2}$ which cannot be simultaneous solvents of any QME and two matrices $S_{1}^{\prime}$ and $S_{2}^{\prime}$ which are simultaneous solvents of infinitely many QME.

We underline that eqs. (8) and (9) may be indirectly constructed exploiting lemma 2.14 of ref.Gohberg 1982. 


\section{Symmetric functions associated to a QME}

\subsection{Elementary symmetric functions associated to a QME}

It is interesting to point out that under the condition of validity of eqs. (8) and (9), the further assumption that $\left[S_{1}, S_{2}\right]=0$ leads us, as expected, to the well known elementary relations $\mathcal{L}_{0}=-S_{1} S_{2}$ and $\mathcal{L}_{1}=S_{1}+S_{2}$ which in turn implies that $\left[\mathcal{L}_{0}, \mathcal{L}_{1}\right]=0$. Even if we are sure that, being in addition $\Delta=\left(S_{1}-S_{2}\right)^{2}, \sqrt{\Delta}$ exists, we do not fully recover all the properties holding in the scalar case since, as previously noted, there might exist matrix values of $\sqrt{\Delta}$ not commuting with $\mathcal{L}_{1}$. The two expressions $S_{1}+S_{2}$ and $S_{1} S_{2}$ play an important role in the context of the theory of quadratic scalar equations. They indeed are the two associated elementary symmetric functions of the two (only and always existing) roots of the QSE, in terms of which every rational symmetric function of the same roots $S_{1}$ and $S_{2}$ is rationally expressible. It is immediate to persuade ourselves that for a QME admitting at least the two solvents $S_{1}$ and $S_{2}$, matrix functions like $S_{1}+S_{2}$ and $S_{1} S_{2}$, although the simplest ones possessing permutational invariance under the exchange of $S_{1}$ with $S_{2}$, cannot occupy a special place for the QME simply because they are not invariant in the set $\mathcal{S}$ of all the possible solvents of the QME. This means that in general $S_{1}+S_{2} \neq S_{1}^{\prime}+S_{2}^{\prime}$ and/or $S_{1} S_{2} \neq S_{1}^{\prime} S_{2}^{\prime}$ with $S_{1}, S_{2}, S_{1}^{\prime}, S_{2}^{\prime} \in \mathcal{S}$ so that these functions are not biunivocally determined by the coefficients of the QME under scrutiny. Theorem 1 suggests to identify the right hand sides of eq. (8) and (9) as the elementary symmetric functions of two solvents to be used when dealing with a QME. These expressions indeed are not only permutationally invariant but their matrix values do not change when we substitute a complete pair of solvents $S_{1}, S_{2}$ with another one complete too. For this reason we are stimulated to introduce the following

Definition 1 Let $S_{1}$ and $S_{2}$ be a complete pair of solvents of a $Q M E$ (1). The two expressions

$$
\Sigma_{2}\left(S_{1}, S_{2}\right)=\left(S_{1}^{2}-S_{2}^{2}\right)\left(S_{1}-S_{2}\right)^{-1}
$$

and

$$
\Pi_{2}\left(S_{1}, S_{2}\right)=S_{1}^{2}\left(S_{1}-S_{2}\right)^{-1} S_{2}-S_{2}^{2}\left(S_{1}-S_{2}\right)^{-1} S_{1}
$$

are called elementary symmetric functions associated to a QME.

\subsection{Symmetric functions associated to a $\mathrm{QME}$}

It is noteworthy to observe that for a given QME, simple permutationally invariant expressions like $\mathbf{S}_{p, 2}=S_{1}^{p}+S_{2}^{p}$ or $\mathbf{\Pi}_{p, 2}=S_{1}^{p} S_{2}+S_{2}^{p} S_{1}$ with $p=1,2, \ldots$, generally lacking of invariance in $\mathcal{S}$ whatever $p$ is, cannot be in general expressed in terms of $\mathcal{L}_{0}$ and $\mathcal{L}_{1}$. We, thus, claim that it 
is not true that every symmetric function of two solvents of a QME not possessing permutational invariance in $\mathcal{S}$, is rationally expressible in terms of the elementary symmetric functions associated to a QME (14) and (36) only. In particular, this means that even $\mathbf{S}_{1,2}=S_{1}+S_{2}$ and $\mathbf{\Pi}_{1,2}=S_{1} S_{2}+$ $S_{2} S_{1}$ suffer such a difficulty and for this reason we call them irreducible symmetric functions. We are however going to show that when $S_{1}$ and $S_{2}$ constitute a complete pair of solvents of a QME, then $\mathbf{S}_{p, 2}$ and $\boldsymbol{\Pi}_{p, 2}$ may be represented in terms of the elementary symmetric functions $\Sigma_{2}\left(S_{1}, S_{2}\right)$ and $\Pi_{2}\left(S_{1}, S_{2}\right)$ given by eqs. (14) and (36) respectively as well as in terms of the irreducible expressions $\mathbf{S}_{1,2}$ and $\boldsymbol{\Pi}_{1,2}$ respectively. We anticipate that such a representation of $S_{p, 2}$ may be interpreted as a generalization of the Waring formula, well-known in the context of QSE.

To this end we exploit a recently published result concerning the exact resolution of the matrix Cauchy problem

$$
\left\{\begin{array}{c}
Y_{p+2}=\mathcal{L}_{0} Y_{p}+\mathcal{L}_{1} Y_{p+1} \\
Y_{0}=0, \quad Y_{1}=B
\end{array}\right.
$$

where the variable $p$ runs in $\mathbb{N}$ and $Y_{p}, \mathcal{L}_{1}$ and $\mathcal{L}_{0}$ belong to $M_{n}(\mathbb{C})$ (Jivulescu et al. 2007). We have been able to extend the successful technique envisaged in this reference to solve the more general Cauchy problem

$$
\left\{\begin{array}{r}
Y_{p+2}=\mathcal{L}_{0} Y_{p}+\mathcal{L}_{1} Y_{p+1} \\
Y_{0}=A, \quad Y_{1}=B
\end{array}\right.
$$

getting the following always existing unique matrix solution

$$
Y_{p}=\alpha_{p} A+\beta_{p} B
$$

where

$$
\alpha_{p}=\left\{\begin{array}{lll}
\sum_{t=0}^{\left[\frac{p-2}{2}\right]}\left\{\mathcal{L}_{0}^{(t)} \mathcal{L}_{1}^{(p-2-2 t)}\right\} \mathcal{L}_{0} & \text { if } & p \geq 2 \\
0 & \text { if } & p=1 \\
E & \text { if } & p=0
\end{array}\right.
$$

and

$$
\beta_{p}=\left\{\begin{array}{ll}
{\left[\frac{p-1}{2}\right]} & \text { if } \quad p \geq 1 \\
\sum_{t=0}^{(t)}\left\{\mathcal{L}_{0}^{(t)} \mathcal{L}_{1}^{(p-1-2 t)}\right\} & \text { if } \quad p=0
\end{array},\right.
$$

where $E$ is the unitary matrix. The symbol $\left\{\mathcal{L}_{0}^{(u)} \mathcal{L}_{1}^{(v)}\right\}$, introduced in (Jivulescu et al. 2007), denotes the sum of all possible distinct permutations of $u$ factors $\mathcal{L}_{0}$ and $v$ factors $\mathcal{L}_{1}$.

As an immediate application of eqs. (17) and (18), let firstly notice that if $S$ is a solvent of eq. (11), then $S^{2}-\mathcal{L}_{1} S-\mathcal{L}_{0}=0$ and consequently 
$S^{p+2}-\mathcal{L}_{1} S^{p+1}-\mathcal{L}_{0} S^{p}=0$, where $p \in \mathbb{N}$. This means that the $p$-th power of any arbitrary solvent $S$ of eq. (11) satisfies the following Cauchy problem

$$
\left\{\begin{array}{l}
S^{p+2}=\mathcal{L}_{0} S^{p}+\mathcal{L}_{1} S^{p+1} \\
S^{0}=E, \quad S^{1}=S
\end{array}\right.
$$

which, coinciding with that expressed by eq. (17), leads to the following formula

$$
S^{p}=\beta_{p} S+\alpha_{p} E,
$$

It is worth noticing that the two operator coefficients $\alpha_{p}$ and $\beta_{p}$ appearing in the right hand side of eq. (22) are completely determined by the knowledge of the coefficients $\mathcal{L}_{0}$ and $\mathcal{L}_{1}$ only. Thus, eq. (22) can be regarded as an effective linearization of $S^{p}$, for any solvent $S$, becoming indeed a computational tool in the sense that the expressions $\alpha_{p}$ and $\beta_{p}$, for a given $p$, may be calculated only once, whatever the solvent $S$ is.

In passing we observe that if $S$ represents the transfer matrix of a "box" then, whatever its nature is, $S^{p}$ may be interpreted as the output after $p$ identical iterations, the QME admitting $S$ as solvent implicitly defining the transmission features of the box. In the last section we will exploit this point of view to deduce the transfer matrix of an $N$-period potential.

Eq. (22) can be generalized. Given indeed $r$-simultaneous solvents $S_{1}, S_{2}, \ldots, S_{r}$ of eq. (1), let's introduce

$$
\mathbf{S}_{p, r}=\sum_{i=1}^{r} S_{i}^{p}
$$

a permutationally invariant quantity under the exchange of any $i$ with any $j,(i, j=1, \ldots, r)$. It is immediate to persuade oneself that such a quantity satisfies the Cauchy problem

$$
\left\{\begin{aligned}
\mathbf{S}_{p+2, r} & =\mathcal{L}_{0} \mathbf{S}_{p, r}+\mathcal{L}_{1} \mathbf{S}_{p+1, r} \\
\mathbf{S}_{0, r} & =r E, \quad \mathbf{S}_{1, r}=\sum_{i=1}^{r} S_{i}
\end{aligned}\right.
$$

which, in view of eq. (18), admits the solution

$$
\mathbf{S}_{p, r}=\beta_{p}\left(\sum_{i=1}^{r} S_{i}\right)+r \alpha_{p} E
$$

This formula is at glance deducible from eq. (22) which in turn is recoverable from eq. (25) simply putting $r=1$. We emphasize that arbitrarily fixing $p$, the expression of $\mathbf{S}_{p, r}$ given by eq. (25) is linearly related to $\mathbf{S}_{1, r}$ in the sense that the operator coefficients $\alpha_{p}$ and $\beta_{p}$ are independent both on the number $r$ of solvents involved and on the group of $r$-solvents chosen in the set $\mathcal{S}$ of all solvents of eq. (1). 
We recall that the set $\mathcal{S}$ may be also infinite thus permitting us to chose in infinitely many ways the family of $r$-solvents satisfying eq. (25). In addition, like eq. (22), this equation may be regarded as a computational tool for the $p$-power sum of $r$ solvents of eq. (11).

We wish to notice that putting $p=r=2$ into eq. (25), under the condition that $S_{1}$ and $S_{2}$ are a complete pair of simultaneous solvents of eq. (11), yields

$$
\mathbf{S}_{2,2}=\Sigma_{2}\left(S_{1}, S_{2}\right) \mathbf{S}_{1,2}-2 \Pi_{2}\left(S_{1}, S_{2}\right) E
$$

which may be interpreted as generalization to the matrix case of the classical Girard- Newton formula for symmetric polynomials in two scalar variables (Sansone 1952).

In the same spirit and hypothesis we are also able to get an extension to the matrix case of the well-known Waring formula for two variables. Putting indeed $r=2$ into the identity (25), leaving instead the integer $p$ free to run in $\mathbb{N}$, we immediately get

$$
\mathbf{S}_{p, 2}=\beta_{p}\left(S_{1}+S_{2}\right)+2 \alpha_{p} E
$$

The peculiar feature of eq. (27) is that the presence of the operators $\alpha_{p}$ and $\beta_{p}$ amounts at rewriting the $p$-th power sum $\mathbf{S}_{p, 2}$ in terms of $\Sigma_{2}\left(S_{1}, S_{2}\right), \Pi_{2}\left(S_{1}, S_{2}\right)$, the above defined elementary symmetric functions associated to the QME, for this reason we claim that eq. (27) may be interpreted as generalization to the matrix case of the Waring formula for two scalar variables (Sansone 1952) .

Another direct consequence of eq. (17) concerns the permutationally invariant quantity

$$
\Pi_{p, r}=\sum_{1 \leq i<j \leq r}\left(S_{i}^{p} S_{j}+S_{i} S_{j}^{p}\right)
$$

It is not difficult to see that the quantity $\left(S_{i}^{p} S_{j}+S_{j}^{p} S_{i}\right)$, for any given $i$ and $j$, satisfies eq. (22), that is

$$
S_{i}^{p} S_{j}+S_{j}^{p} S_{i}=\beta_{p}\left(S_{i} S_{j}+S_{j} S_{i}\right)+\alpha_{p}\left(S_{i}+S_{j}\right)
$$

from which we immediately deduce that

$$
\boldsymbol{\Pi}_{p, r}=\beta_{p} \boldsymbol{\Pi}_{1, r}+(r-1) \alpha_{p} \mathbf{S}_{1, r}
$$

Applying to eq. (30) the same arguments used to deduce eq. (27) from eq. (25) immediately yields

$$
\boldsymbol{\Pi}_{p, 2}=\alpha_{p} \mathbf{S}_{1,2}+\beta_{p} \boldsymbol{\Pi}_{1,2}
$$

which is of relevance since it expresses $\boldsymbol{\Pi}_{p, 2}$ in terms of the irreducible expressions $\mathbf{S}_{1,2}=\left(S_{1}+S_{2}\right)$ and $\boldsymbol{\Pi}_{1,2}=S_{1} S_{2}+S_{2} S_{1}$ and the elementary symmetric 
functions associated to the QME $\Sigma_{2}\left(S_{1}, S_{2}\right), \Pi_{2}\left(S_{1}, S_{2}\right)$, considering that $\alpha_{p}$ and $\beta_{p}$ are functions of $\mathcal{L}_{0}=\Sigma_{2}\left(S_{1}, S_{2}\right)$ and $\mathcal{L}_{1}=-\Pi_{2}\left(S_{1}, S_{2}\right)$.

It is interesting to stress that the unavoidable presence of $\mathbf{S}_{1,2}$ in eq. (27) and of $\boldsymbol{\Pi}_{1,2}$ too into eq. (31) does not allow to write down the permutationally invariant expressions $\mathbf{S}_{p, 2}$ and $\boldsymbol{\Pi}_{p, 2}$ in terms of the noncommutative coefficients $\mathcal{L}_{0}$ and $\mathcal{L}_{1}$ only. This observation confirms our claim that the fundamental theorem regarding the symmetric functions of two scalar variables does not hold in the matrix case.

The elementary symmetric functions associated to the QME (1) may be easily generalized. To this end let's write eq. (22) for both elements $S_{1}$ and $S_{2}$ of a complete pair of simultaneous solvents

$$
\left\{\begin{array}{l}
S_{1}^{p}=\beta_{p} S_{1}+\alpha_{p} \\
S_{2}^{p}=\beta_{p} S_{2}+\alpha_{p}
\end{array}\right.
$$

It is simple to solve this system of equations in the unknowns $\alpha_{p}$ and $\beta_{p}$ obtaining

$$
\alpha_{p}=S_{2}^{p}\left(S_{1}-S_{2}\right)^{-1} S_{1}-S_{1}^{p}\left(S_{1}-S_{2}\right)^{-1} S_{2}
$$

and

$$
\beta_{p}=\left(S_{1}^{p}-S_{2}^{p}\right)\left(S_{1}-S_{2}\right)^{-1}
$$

Eqs. (33) and (34) reduce to $-\Pi_{2}\left(S_{1}, S_{2}\right)$ and $\Sigma_{2}\left(S_{1}, S_{2}\right)$ when $p=2$. In addition, the right hand side of both equations are certainly invariant in the set $\mathcal{S}$. For this reason, we are induced to introduce the following

Definition 2 Let $S_{1}$ and $S_{2}$ be a complete pair of simultaneous solvents of a $Q M E$ (1). The two expressions

$$
\Sigma_{p}\left(S_{1}, S_{2}\right)=\left(S_{1}^{p}-S_{2}^{p}\right)\left(S_{1}-S_{2}\right)^{-1}
$$

and

$$
\Pi_{p}\left(S_{1}, S_{2}\right)=S_{1}^{p}\left(S_{1}-S_{2}\right)^{-1} S_{2}-S_{2}^{p}\left(S_{1}-S_{2}\right)^{-1} S_{1}
$$

are called symmetric functions associated to a $Q M E$.

It is of relevance that $\Sigma_{p}\left(S_{1}, S_{2}\right)$ and $\Pi_{p}\left(S_{1}, S_{2}\right)$ may be expressed in terms of elementary symmetric functions associated to a QME $\Sigma_{2}\left(S_{1}, S_{2}\right)$ and $\Pi_{2}\left(S_{1}, S_{2}\right)$ as easily seen considering the definition of $\alpha_{p}$ and $\beta_{p}$ and in the view of the eqs. (8) and (9). If we restrict our definition of symmetric functions associated to a QME to all those expressions of two complete solvents satisfying the condition to be permutationally invariant into $\mathcal{S}$ then such expressions like (35) and (36) must be expressible in terms of $\mathcal{L}_{0}$ and $\mathcal{L}_{1}$ and then in terms of $\Sigma_{2}\left(S_{1}, S_{2}\right)$ and $\Pi_{2}\left(S_{1}, S_{2}\right)$.

Thus, we claim that any symmetric function associated to a QME may be written down in terms of elementary symmetric functions. 


\section{A simple application}

If the order of the unknown matrix $X$ of eq. (11) is fixed at its minimum value 1 we recover the scalar case getting simultaneously rid of both the matrix nature of the problem and of any complication stemming from the noncommutativity between $\mathcal{L}_{0}$ and $\mathcal{L}_{1}$ as well as between $X$ and the two coefficients. A simpler but not trivial problem, someway intermediate between the full matrix noncommutative eq. (11) and its scalar version, may be singled out requiring solely the commutativity between the two coefficients $\mathcal{L}_{0}$ and $\mathcal{L}_{1}$. We emphasize that such a restriction does not spoil eq. (11) of interest enabling us, in turn, to bring to light some peculiar properties consequent to its matrix nature only. We stress indeed that on the basis of the results reported in the previous sections of this paper, the hypothesis $\left[\mathcal{L}_{0}, \mathcal{L}_{1}\right]=0$ does not lead to any effective recipe to build up the set of solvents of eq. (1) which may be still empty or contain finitely-many or infinitely many elements. The concrete advantage provided by the condition $\left[\mathcal{L}_{0}, \mathcal{L}_{1}\right]=0$ is that it makes the matrix expressions of $\alpha_{p}$ and $\beta_{p}$ algebraically manageable disclosing their connection with the Chebyshev polynomials of the second kind $\mathcal{U}_{p}(x)$ (Murray-Spiegel 1998) defined as follows

$$
\mathcal{U}_{p}[x]=\sum_{m=0}^{[p / 2]}(-1)^{m} \frac{(p-m) !}{m !(p-2 m) !}(2 x)^{p-2 m}
$$

Taking indeed into consideration the commutativity between $\mathcal{L}_{0}$ and $\mathcal{L}_{1}$ as well as the fact that the number of all the different terms appearing in the operator symbol $\left\{\mathcal{L}_{0}^{(u)} \mathcal{L}_{1}^{(v)}\right\}$ coincides with the binomial coefficient $\left(\begin{array}{c}u+v \\ m\end{array}\right)$, with $m=\min (u, v)$, we may rewrite for $p \geq 2$ that

$$
\alpha_{p}=\sum_{t=0}^{[(p-2) / 2]}\left(\begin{array}{c}
p-2-t \\
t
\end{array}\right) \mathcal{L}_{0}^{t+1} \mathcal{L}_{1}^{p-2-2 t}
$$

while $\alpha_{0}=E$ and $\alpha_{1}=0$. Assuming the existence of the inverse of the square root of $\mathcal{L}_{0}$ opens the way to express $\alpha_{p}$ as

$$
\alpha_{p}=-\left(-\mathcal{L}_{0}\right)^{p / 2} \sum_{t=0}^{[(p-2) / 2]}(-1)^{t}\left(\begin{array}{c}
p-2-t \\
t
\end{array}\right)\left(\mathcal{L}_{1}\left(-\mathcal{L}_{0}\right)^{-\frac{1}{2}}\right)^{(p-2-2 t)}
$$

that is

$$
\alpha_{p}=-\left(-\mathcal{L}_{0}\right)^{\frac{p}{2}} \mathcal{U}_{p-2}\left[\frac{1}{2} \mathcal{L}_{1}\left(-\mathcal{L}_{0}\right)^{-\frac{1}{2}}\right], \quad p \geq 2
$$

Following a similar procedure and under the same hypothesis we have 


$$
\begin{gathered}
\sum_{t=0}^{[(p-1) / 2]}\left(\begin{array}{c}
p-1-t \\
t
\end{array}\right) \mathcal{L}_{0}^{t} \mathcal{L}_{1}^{p-1-2 t}= \\
\left(-\mathcal{L}_{0}\right)^{(p-1) / 2} \sum_{t=0}^{[(p-1) / 2]}(-1)^{t}\left(\begin{array}{c}
p-1-t \\
t
\end{array}\right)\left(\mathcal{L}_{1}\left(-\mathcal{L}_{0}\right)^{-\frac{1}{2}}\right)^{(p-1-2 t)}
\end{gathered}
$$

from which

$$
\beta_{p}=\left(-\mathcal{L}_{0}\right)^{\frac{p-1}{2}} \mathcal{U}_{p-1}\left[\frac{1}{2} \mathcal{L}_{1}\left(-\mathcal{L}_{0}\right)^{-\frac{1}{2}}\right], \quad p \geq 1
$$

while $\beta_{0}=0$. Inserting eqs. (40) and (41) into eq. (22) yields

$S^{p}=\left(-\mathcal{L}_{0}\right)^{\frac{p-1}{2}} \mathcal{U}_{p-1}\left[\frac{1}{2} \mathcal{L}_{1}\left(-\mathcal{L}_{0}\right)^{-\frac{1}{2}}\right] S-\left(-\mathcal{L}_{0}\right)^{\frac{p}{2}} \mathcal{U}_{p-2}\left[\frac{1}{2} \mathcal{L}_{1}\left(-\mathcal{L}_{0}\right)^{-\frac{1}{2}}\right] E, \quad p \geq 2$

which reduces to the trivial identities $S=S$ and $E=E$, when $p=1$ and $p=0$ respectively.

If the two coefficients $\mathcal{L}_{0}$ and $\mathcal{L}_{1}$ appearing in eq. (22) commute, then the right hand side of formula (42) provides the definition of the transfer matrix of a single "equivalent box" substituting all the original $p$ ones.

It is worth noting that the practical use of eq. (42) is greatly facilitated when the minimal polynomial of $S$ has a degree 2 . We remind that the monic polynomial of minimum degree $p(x)$ such that $p(S)$ is the zero matrix is called the minimal polynomial of $S$ and that it always exists and is unique (Gantmacher 1998). Let us suppose that the distinct roots of the characteristic polynomial of $S$, a given solvent of eq. (11), are only two and denote them by $\lambda_{1}$ and $\lambda_{2}$. If in addition we stipulate that $S$ is diagonalizable, it is well-known that, then, its minimal polynomial coincides with the characteristics polynomial of the auxiliary two by two diagonal matrix $\widetilde{S}=\left(\begin{array}{cc}\lambda_{1} & 0 \\ 0 & \lambda_{2}\end{array}\right)$. This amounts at claiming that $S$ is also solvent of the QME $S^{2}-(\operatorname{tr} \widetilde{S}) S+(\operatorname{det} \widetilde{S}) E=0$, relatively simpler than eq. (1) since $\mathcal{L}_{0}$ and $\mathcal{L}_{1}$ are now proportional to $E$. As a direct consequence formula (42) may be easily exploited to find $S^{p}$, for any $p$. We underline that, when $S$ is of order two, the applications of this formula may be made systematically easier, simply having recourse to the Cayley-Hamilton identity $S^{2}-(\operatorname{tr} S) S+(\operatorname{det} S) E=0$. Quite recently, for example, this approach has been used to express the $N$ - period transfer matrix $M^{N}$ of a photonic crystal as a linear function of the unit cell-matrix $M=\left(\begin{array}{cc}1 / t & r^{*} / t^{*} \\ r / t & 1 / t^{*}\end{array}\right)$, where $t$ and $r$ are the complex transmission and reflection coefficients of the unit cell respectively, satisfying the condition $|r|^{2}+|t|^{2}=1$ (Benickson et al. 1996). It is easy to persuade ourselves that the Cayley-Hamilton theorem leads in such a case to the following simple QME

$$
M^{2}-2 \cos \beta M+E=0
$$


where $\cos \beta=\operatorname{Re}(1 / t)$.

Thus, resorting to eq. (42), we may soon establish the transfer-matrix reduction identity

$$
M^{N}=\mathcal{U}_{N-1}[\cos \beta] M-\mathcal{U}_{N-2}[\cos \beta] E
$$

which may be put into the following closed form (Murray-Spiegel 1998)

$$
M^{N}=\frac{\sin N \beta}{\sin \beta} M-\frac{\sin (N-1) \beta}{\sin \beta} E
$$

This expression coincides with that differently derived in ref. (Bendickson et al. 1996).

\section{Conclusive remarks}

In this paper we have studied the quadratic matrix equation $X^{2}-\mathcal{L}_{1} X-$ $\mathcal{L}_{0}=0$ bringing to light new peculiar properties of its, clearly traceable back to the inherent noncommutative character of the problem. Driven by the knowledge of any aspect concerning the simple classical theory of the scalar quadratic equations, we have formulated and coped with some basic questions putting into evidence remarkable differences between the matrix and the scalar case. Exploring the meaning and the consequences of such differences we have succeeded in introducing the definition and the explicit expressions of the matrix elementary symmetric functions associated to a QME by which some notable identities involving solvents of the QME have been derived.

Other questions greatly stimulate our attention like for example the notion of multiple solvents or how to define a factorization procedure, if any, of a quadratic matrix polynomial from the knowledge of its finitely-many or infinitely-many solvents. We believe that to develop the theory of the matrix algebraic equations, quadratic or of higher order, might provide useful tools to overcome technical mathematical obstacles encountered in the resolution of physical problems both in a classical and in a quantum context.

\subsection{Acknowledgments}

The authors would like to thank professor A. Giambruno, dr. A. Settimi, dr. B. Militello and dr. R. Messina for fruitful discussions.

\section{A APPENDIX}

Let's give few examples clearly illustrating our affirmation that the fundamental theorem of algebra does not hold for matrix equation; thus, our examples point out QMEs having no solvent or infinitely many solvents as 
well as equations with one or finitely-many solvents. In fact, these different scenarios are mainly traceable back to the existence of the square root of a $n \times n$ matrix, say $M$, which is a multivalued operation generally not representable in the form of a polynomial in $M$ (Horn 1999).

Firstly, let's consider the quadratic matrix equation $X^{2}-2 E X-\mathcal{L}_{0}=0$, where $\mathcal{L}_{0}$ is the square matrix of order $n$ with its diagonal entries equal to -1 , the elements of the first superdiagonal equal to unit and all the other vanishing. This equation may be immediately written as $(X-E)^{2}=\mathcal{L}_{0}+E$ and it does not admit solvents since the square roots of $\mathcal{L}_{0}+E \equiv H^{(n)}$ does not exist (Gantmacher 1998).

The matrix equation $X^{2}+E X=\mathcal{L}_{0}$ with $\mathcal{L}_{0} n \times n$ diagonal matrix having $\left(\mathcal{L}_{0}\right)_{i i}=2 \delta_{i, 1}$ admits finitely-many diagonal solvents in the form

$$
S_{1}=\left\{1, \epsilon_{2}, \epsilon_{3}, \ldots, \epsilon_{n}\right\}, \quad S_{-2}=\left\{-2, \nu_{2}, \nu_{3}, \ldots, \nu_{n}\right\}
$$

where each of the diagonal entries $\epsilon_{2}, \epsilon_{3}, \ldots, \epsilon_{n}$ in $S_{1}$ and $\nu_{2}, \nu_{3}, \ldots, \nu_{n}$ in $S_{-2}$ may arbitrarily assume the values 0 or -1 .

Finally, the matrix equation $X^{2}-E X=2 E$ provides an example wherein infinitely-many solvents exist having the form of lower triangular matrices

$$
\begin{aligned}
X & =\left(\begin{array}{cccccc}
-1 & & & & & \\
c_{1} & 2 & & & & \\
0 & 0 & -1 & & & \\
c_{2} & & & 2 & & \\
\cdots & & & & \\
c_{n / 2} & & & -1 & \\
0 & & & & 2
\end{array}\right)_{n-\text { even }}, \\
X & =\left(\begin{array}{cccccc}
-1 & & & & & \\
c_{1} & 2 & & & & \\
0 & 0 & -1 & & & \\
c_{2} & & & 2 & & \\
\cdots & & & & & \\
c_{n-1 / 2} & & & & \\
0 & & & & -1
\end{array}\right)_{n-\text { odd }}
\end{aligned}
$$

where all the non-indicated entries are equal to 0 and $c_{1}, c_{2}, \ldots, c_{\frac{n}{2}}\left(c_{\frac{n-1}{2}}\right) \in$ $\mathbb{C}$ 


\section{B Appendix}

The $n$-quadratic matrices

$S_{1}=\left(\begin{array}{ccccc}1 & 2 & 2 & \ldots & 2 \\ 0 & 1 & 2 & \ldots & 2 \\ \ldots & \ldots & \ldots & \ldots & \ldots \\ 0 & 0 & 0 & \ldots & 1\end{array}\right), S_{2}=\left(\begin{array}{ccccc}-1 & 0 & 0 & \ldots & 0 \\ -2 & -1 & 0 & \ldots & 0 \\ \ldots & \ldots & \ldots & \ldots & \ldots \\ -2 & -2 & -2 & \ldots & -1\end{array}\right)$

cannot be both solvents of the same QME due to fact that the matrix equation which furnishes the coefficient $\mathcal{L}_{1}$

$$
\mathcal{L}_{1}\left(S_{1}-S_{2}\right)=\left(S_{1}^{2}-S_{2}^{2}\right)
$$

admits no solution. The incompatibility of equation is based on the RoucheCapelli's theorem because $\operatorname{rank}\left(S_{1}-S_{2}\right) \neq \operatorname{rank}\left(S_{1}^{2}-S_{2}^{2}\right)$, where

$$
\begin{aligned}
& S_{1}-S_{2}=\left(\begin{array}{ccccc}
2 & 2 & 2 & \ldots & 2 \\
2 & 2 & 2 & \ldots & 2 \\
\ldots & \ldots & \ldots & \ldots & \ldots \\
2 & 2 & 2 & \ldots & 2
\end{array}\right) \text {, } \\
& S_{1}^{2}-S_{2}^{2}=\left(\begin{array}{ccccc}
0 & 1 \cdot 2^{2} & 2 \cdot 2^{2} & \ldots & (n-1) \cdot 2^{2} \\
-1 \cdot 2^{2} & 0 & 1 \cdot 2^{2} & \ldots & (n-2) \cdot 2^{2} \\
\ldots & \ldots & \ldots & \ldots & \ldots \\
-(n-1) \cdot 2^{2} & -(n-2) \cdot 2^{2} & -(n-3) \cdot 2^{2} & \ldots & 0
\end{array}\right)
\end{aligned}
$$

Further, the $n$ - quadratic matrices

$$
S_{1}^{\prime}=\left(\begin{array}{ccccc}
1 & 0 & \ldots & 0 & 0 \\
0 & 1 & \ldots & 0 & 0 \\
\ldots & \ldots & \ldots & \ldots & \ldots \\
0 & 0 & \ldots & 1 & 0 \\
0 & 0 & \ldots & 0 & 3
\end{array}\right), S_{2}^{\prime}=\left(\begin{array}{ccccc}
1 & 0 & \ldots & 0 & 0 \\
0 & 1 & \ldots & 0 & 0 \\
\ldots & \ldots & \ldots & \ldots & \ldots \\
0 & 0 & \ldots & 1 & 0 \\
0 & 0 & \ldots & 0 & 2
\end{array}\right)
$$

are two solvents of the class of the QME for which

$$
\begin{array}{r}
\mathcal{L}_{1}=\left(\begin{array}{ccccc}
a_{11} & a_{12} & \ldots & a_{1 n-1} & 0 \\
a_{21} & a_{22} & \ldots & a_{2 n-1} & 0 \\
\ldots & \ldots & \ldots & \ldots & \ldots \\
a_{n-11} & a_{n-12} & \ldots & a_{n-1 n-1} & 0 \\
a_{n 1} & a_{n 2} & \ldots & a_{n n-1} & 5
\end{array}\right), \\
\mathcal{L}_{0}=\left(\begin{array}{ccccc}
1-a_{11} & -a_{12} & \ldots & -a_{1 n-1} & 0 \\
-a_{21} & 1-a_{22} & \ldots & -a_{2 n-1} & 0 \\
\ldots & \ldots & \ldots & \ldots & \ldots \\
-a_{n-11} & -a_{n-12} & \ldots & 1-a_{n-1 n-1} & 0 \\
-a_{n 1} & -a_{n 2} & \ldots & -a_{n n-1} & -6
\end{array}\right), a_{i j} \in \mathbb{C}
\end{array}
$$




\section{References}

[1] Bai Z. Z., Guo X. X. and Yin J. F. 2005 On two iteration methods for the quadratic matrix equations, International Journal of Numerical Analysis and Modeling 2, 114-122

[2] Bendickson J. M., Dowling J. P. 1996 Analytic expressions for the electromagnetic mode density in finite, one-dimensional, photonic bandgap structures Physical Review E 534 4107-4121

[3] Butler G. J., Johnson C. R. and Wolkowics H. 1985 Nonnegative solutions of a quadratic matrix equation arising from comparison theorems in ordinary differential equations SIAM J. Alg. Disc. Meth. 6, no 1, 47

[4] Dennis J. E., Traub J. F. and Weber R. P. 1976 The algebraic theory of matrix polynomials SIAM J. Numer. Anal. 13, 831

[5] Eisenfeld J. 1973 Operator equations and nonlinear eigenparameter problems J. Funct. Anal. 12475

[6] Gantmacher F. R. 1998 The Theory of Matrices (American Mathematical Society, Providence, Rhode Island)

[7] Gelfand I. M., Krob D., Lascoux A., Leclerc B., Retakh V. S. and Thibon J. Y. 1995 Noncommutative symmetric functions Adv. in Math. 112

[8] Giambruno A. and Zaicev M. 2005 Polynomial identities and asymptotic methods (American Mathematical Society)

[9] Gohberg I., Lancaster P. and Rodman L. 1978 Spectral analysis of matrix polynomials I. Canonical forms and Divisors Linear algebra and its applications 20, 1-44

[10] Gohberg I., Lancaster P. and Rodman L. 1978 Spectral analysis of matrix polynomials II. The resolvent form and spectral divisors Linear algebra and its applications 21, 65-88

[11] Gohberg I., Lancaster P. and Rodman L. 1982 Matrix polynomials (New York: Academic)

[12] Gohberg I., Lancaster P. and Rodman L. 2006 Invariant Subspaces of Matrices with Applications (SIAM)

[13] Higham N. J. and Kim H. M. 2000 Numerical analysis of aquadratic matrix equation Journal of Numerical Analysis 20499 
[14] Higham N. J. and Kim H. M. 2001 Solving a quadratic matrix equation by Newton's method with exact line searches SIAM J. Matrix Anal. Appl. 23 no 2303

[15] Higham N. J. 1987 Computing real square roots of a real matrix Linear Algebra Appl. 88/89 405

[16] Horn R and Johnson C 1999 Topics in Matrix Analysis (Cambridge University Press)

[17] Jivulescu M. A., Messina A. and Napoli A. 2007 Exact treatment of linear difference equations with noncommutative coefficients Mathematical Methods in Applied Sciences 302147

[18] Krein M. G. and Langer H. 1978 On some mathematical principles in the linear theory of damped oscillations of continua I Integral Equations and Operator Theory 1/3, 364-399

[19] Lancaster P. 1966 Lambda-Matrices and Vibrating Systems (Oxford: Pergamon)

[20] Lancaster P. and Rokne J. G. 1977 Solutions of nonlinear operator equations SIAM J. Math. Anal. 8, 448-457

[21] McFarland J. E. 1958 An iterative solution of the quadratic equation in Banach space Proc. Am. Math. Soc 9, 824-830

[22] Merzbacher E 1968 Matrix methods in quantum mechanics American Journal of Physics vol $\mathbf{3 6}$ no. 9

[23] Murray R. Spiegel 1998 Schaum's Mathematical Handbook of Formulas and Tables (McGraw-Hill )

[24] Sansone G 1952 Lezioni di analisi matematica CEDAM vol 1

[25] Shurbet G. L., Lewis T. O. and Boullion L. 1974 Quadratic matrix equations The Ohio Journal of Science $\mathbf{7 4}$ no 5273 